Journal of Eye Movement Research

$6(4): 5,1-11$

\title{
Combining EEG and Eye Tracking: Using Fixation-Locked Potentials in Visual Search
}

\author{
Brent Winslow \\ Design Interactive, Inc. \\ Angela Carpenter \\ Jesse Flint \\ Design Interactive, Inc. \\ Design Interactive, Inc. \\ Xuezhong Wang \\ Design Interactive, Inc. \\ David Tomasetti \\ Matthew Johnston \\ Kelly Hale \\ Design Interactive, Inc. \\ Design Interactive, Inc. \\ Design Interactive, Inc.

\begin{abstract}
Visual search is a complex task that involves many neural pathways to identify relevant areas of interest within a scene. Humans remain a critical component in visual search tasks, as they can effectively perceive anomalies within complex scenes. However, this task can be challenging, particularly under time pressure. In order to improve visual search training and performance, an objective, process-based measure is needed. Eye tracking technology can be used to drive real-time parsing of EEG recordings, providing an indication of the analysis process. In the current study, eye fixations were used to generate ERPs during a visual search task. Clear differences in ERPs were observed following training, suggesting that neurophysiological signatures could be developed to prevent errors in visual search tasks.
\end{abstract}

Keywords: Electroencephalography, saccades; visual attention; ERPs

\section{Introduction}

Visual search consists of finding a target in the midst of distractors, and is a complex task that involves many neural pathways and systems including the visual system, working memory, and attention to identify relevant areas of interest within a visual scene (Kastner \& Ungerleider, 2000; Petersen \& Posner, 2012). Visual search has been described both in terms of exogenous and endogenous components. Exogenous visual search is driven by properties of a visual scene, which appear more salient due to human visual processing neural pathways, as the central nervous system is structured to respond to certain stimuli preferentially (Albright, 2012). Visual receptors and pathways have evolved to capture key features automatically, such as color, motion and edge. This automatic, pre-attentive process (Treisman, 2006) is quick (Montagna, Pestilli, \& Carrasco, 2009), and requires little conscious effort. Theories of exogenous control assume a saliency map (Koch \& Ullman, 1985; Treisman \& Gelade, 1980) where locations of likely relevance are identified. Driven by these maps, attention serves as a control system that biases the filtering of feature and location information to support threat detection and response selection (Müller \& Krummenacher, 2006). On the other hand, endogenous, top-down attentional orienting during visual search occurs when attention is consciously directed in a voluntary way according to goals and intentions (Mulckhuyse \& Theeuwes, 2010). Endogenous attention can be allocated to a location within about $300-500 \mathrm{~ms}$ and may be sustained for several seconds (Montagna, et al., 2009). 
Journal of Eye Movement Research $6(4): 5,1-11$
Winslow, B., Carpenter, A., Flint, J., Wang, X., Tomasetti, D., Johnston, M. \& Hale, K (2013) Combining EEG and Eye Tracking: Using Fixation-Locked Potentials in Visual Search
Visual search is the primary role of The Transportation Safety Administration (TSA) Transportation Safety Officers (TSOs), who are tasked with identifying potential threat items within cluttered carry-on bags at over 7000 security checkpoints in the United States. As bags are screened using X-ray technology, TSOs must determine whether they believe the bag to be free from threats, in which the bag is "cleared", whether there is a potential threat, in which the bag is subjected to further search, or whether a serious threat exists. If threats are highly prevalent, potential or serious threat decisions are more likely, and "clear" responses are slower because such a response would often lead to a mistake (Wolfe, Horowitz, \& Kenner, 2005). Baggage screening is a repetitive visual search task that often has a very low probability of encountering a threat, but high consequences if a serious threat is missed. In baggage screening, since threats are of low prevalence, a "cleared" response will often lead to a successful outcome and thus becomes the more frequent decision. Observers will tend to abandon a search in less than the average time required to find a target (Wolfe \& Van Wert, 2010) under such circumstances.

Regardless of the decision made by a TSO, there is little quantifiable information available regarding what led to a decision. The addition of real-time neurophysiological measures could provide a more granular understanding of the decision making process throughout training and performance, and neurophysiological signatures could also be developed to mitigate potential threat misses.

Electroencephalography (EEG) is a well-established non-invasive technique for brain monitoring with high temporal resolution and relatively low cost. As such, EEG has proven to be a critical monitoring and diagnostic tool in the clinic (Lagerlund, Cascino, Cicora, \& Sharbrough, 1996; Mendez \& Brenner, 2006). EEG is also a popular research tool among scientists for evaluating somatosensory responses to stimuli, error detection (Davidson, Jones, \& Peiris, 2007), and sleep or fatigue monitoring (Colrain, 2011; Landolt, 2011), among other uses. Various EEG components in the temporal domain have been used to define distinct phases of cortical processing in response to stimulus presentation. Such event-related potentials (ERPs) have been used to noninvasively study visual (Clark, Fan, \& Hillyard, 1995; Hillyard, Vogel, \& Luck, 1998), auditory (Naatanen \& Picton, 1987), and somatosensory processing (Wada, 1999). One component particularly important in visual processing is the $\mathrm{P} 3$, a time-locked deflection which appears $300-400 \mathrm{~ms}$ after stimulus presentation, first described a half century ago (Sutton, Braren, Zubin, \& John, 1965). Training can alter perception and motor learning (Censor, Sagi, \& Cohen, 2012), including visual discrimination (Stickgold, Whidbee, Schirmer, Patel, \& Hobson, 2000), key to TSO screening tasks, which can be monitored using ERPs (Song, Ding, Fan, \& Chen, 2002). However, the use of such an evaluation outside the laboratory lacks an indication of when a participant visually fixed upon a stimulus of interest.

Eye tracking technology offers the possibility of capturing visual behavior in real-time and monitoring locations of fixations within images (Hansen \& Ji, 2010). Recently, eye tracking technology has become more accurate and user friendly. It has extended to various areas that led to a wide range of applications (Duchowski, 2002; Jacob, 1991). The current study was designed to test the utility of using eye-tracking fixation points on targets to parse simultaneously recorded EEG, and to test the feasibility of developing a unique neurophysiological fixation-locked event related potential (FLERP) classifier to monitor performance in visual search tasks.

\section{Material and methods}

\section{Stimuli and apparatus}

ScreenADAPT software, developed by Design Interactive Inc., is an adaptive software suite designed to reduce the time to criterion baggage screening performance during training, and allows creation of Xray images of carry-on luggage with customized content. The image generator is fed by $\mathrm{X}$-rayed threat and distractor libraries rendered from individual 3D models obtained from public-domain websites, overlaid onto 
Journal of Eye Movement Research $6(4): 5,1-11$
Winslow, B., Carpenter, A., Flint, J., Wang, X., Tomasetti, D., Johnston, M. \& Hale, K (2013) Combining EEG and Eye Tracking: Using Fixation-Locked Potentials in Visual Search 'clear' X-ray bag images in various orientations and positions, where clear bags included a variety of nonthreat items typical of carry-on luggage (see Figure 1). Threat images included guns and knives, and distractor images were chosen to be intentionally similar in size and shape to threats. A single threat or distractor image was inserted into each existing X-ray baggage image.

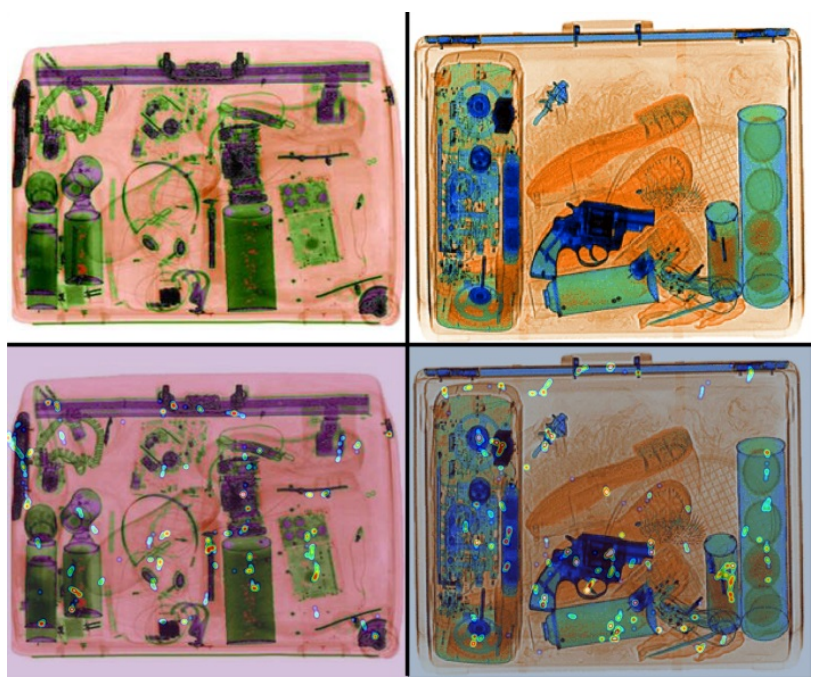

Figure 1 - Example X-ray images of simulated carry-on baggage used in this study. Images were used both without threats (baggage on left), and with specific threats inserted into the images (baggage on right). Gaze locations and durations are shown in a heat map format overlaid on the images in the bottom panels.

\section{Participants}

All methods involving participants were approved by an independent Institutional Review Board. Forty novice participants [20 male, 20 female; average age $28 \pm 8$ (SD) years] completed and received payment of $\$ 100$ USD for participation in this study. All participants were recruited from the community and met minimum recruitment requirements for TSA officers including citizenship (US citizen), age (over 18 years), education (high school diploma or equivalent) and vision (20:20 or corrected to 20:20) requirements. All participants were fully informed about the procedure and purpose of the study, which lasted approximately $2-3$ hours.

\section{Apparatus}

Baggage images were displayed on a $48 \mathrm{~cm}$ flat panel monitor with $1280 \times 1024$ pixels. A standard mouse and keyboard were used to interact with the system. Participants were seated $40 \mathrm{~cm}$ from the monitor, with a remote video eye tracking system (easyGaze, Design Interactive Inc., Oviedo, Florida) situated directly below the monitor at a $30^{\circ}$ viewing angle to acquire participants' eye position. The system utilizes nearinfrared (NIR) LEDs to illuminate the eyes of the participant and gathers the data via binocular dark pupil tracking at $30 \mathrm{~Hz}$. Calibration was done with a 16-point grid to ensure accuracy of both eyes to meet a minimum of $0.5^{\circ}$, which included horizontal and vertical position of the gaze point, distance from each eye to the camera, and pupil diameter. A dispersion-threshold method was used to identify fixations as groups of consecutive gaze points within a maximum separation of 20 pixels (Salvucci \& Goldberg, 2000). Furthermore, a temporal restriction of $100 \mathrm{~ms}$ was applied as the minimum fixation duration to alleviate the device variances.

\section{EEG}

The EEG was recorded throughout the experiment with the Advanced Brain Monitoring (ABM, Carlsbad CA) B-Alert X-10 wireless acquisition system. The system records from $9 \mathrm{Ag}-\mathrm{AgCl}$ electrodes according to the International 10-20 system at Fz, F3, F4, Cz, C3, C4, $\mathrm{POz}, \mathrm{P} 3$ and $\mathrm{P} 4$ at $256 \mathrm{~Hz}$. All electrodes were referenced to additional mastoid electrodes, bandpass filtered at $60 \mathrm{~Hz}$ to remove line noise, and impedances were kept below $40 \mathrm{k} \Omega$. Recorded EEG was decontaminated by removing artifact for EMG, eye blinks, excursions, saturations and spikes by ABM BAlert software. Identification of eye blinks in the EEG is achieved by filtering the fast component of the $\mathrm{Fz}$ channel with a $7 \mathrm{~Hz}$ IIR low-pass filter, applying crosscorrelation analysis to the filtered signal using the positive half of a $40 \mu \mathrm{V} 1.33 \mathrm{~Hz}$ sine wave as the target shape, and applying thresholds to the outputs from the cross-correlation analysis. Minima and maxima analysis in each direction from the point of maximum correlation is used to identify the data points corresponding to the range between the start and end of each eye blink. Once 
Journal of Eye Movement Research $6(4): 5,1-11$
Winslow, B., Carpenter, A., Flint, J., Wang, X., Tomasetti, D., Johnston, M. \& Hale, K (2013) Combining EEG and Eye Tracking: Using Fixation-Locked Potentials in Visual Search eye blink ranges have been determined, the $0.5 \mathrm{~Hz}$ highpass filtered EEG signal from each channel is decontaminated by replacing the data points in the eye blink region with the corresponding data after application of the $4 \mathrm{~Hz}$ filter (Berka, Levendowski, Cvetinovic, Petrovic, Davis, Lumicao, Zivkovic, Popovic, \& Olmstead, 2004).

\section{Visual search scenarios}

Following the donning of the EEG headset and eyetracker calibration, participants were given written instructions that outlined what constituted a threat for this experiment, as well as instructions on how to operate the software, followed by a practice session. The practice session consisted of four trial images, two of which contained threats. Once participants completed the practice session, the experimental session started, which consisted of a pre-test session, and seven additional test sessions interspersed with training sessions. Each test and training session consisted of 64 baggage images with an equal distribution of threat and distractor stimuli. Participants were instructed to scan each bag for threat items, and to complete the task as accurately and quickly as possible. If the participant perceived a threat, they were instructed to click directly on threats with a computer mouse. If the participant did not perceive a threat, they were instructed to press the space bar to "Clear" the bag and move to the next image. Training sessions between each test session were identical to test sessions except EEG and eye tracking data were not gathered, and participants were given performance feedback. Hit rate was defined as the number of hits divided by the sum of hits and misses; miss rate was the number of misses divided by the sum of hits and misses. False alarm (FA) rate was defined as the number of FA divided by the sum of FA and correct rejections (CR); and the $\mathrm{CR}$ rate was defined as the number of $\mathrm{CR}$ divided by the sum of FA and CR. Trials with response times exceeding 2 SD from the mean were discarded.

\section{FLERP analysis}

EEG and eye-tracking synchronization was accomplished via post-processing in MATLAB (Mathworks, Natick MA). Since both the eye tracker and the EEG were run on the same PC, the reported timestamps for both systems queried the same system clock at the ms level when reporting values. The first fixation on a threat/distractor was used to mark the beginning of an event-related potential (ERP) within EEG data for each session. Following the participant response, these time points were classified as hit (threat present, participant clicked on threat), miss (threat present but participant indicated no threat, type II error), FA (no threat present but participant indicates threat in image, type I error) or false positive, and CR (no threat present and participant indicates no threat) (Macmillan \& Creelman, 2004; Wickens, Hollands, Banbury, \& Parasuraman, 2012), as shown in Figure 2. If threats or distractors were not fixated upon, trials were excluded from FLERP analysis. EEGLAB and ERPLAB packages (Delorme \& Makeig, 2004) were used in MATLAB to process the EEG data. After the decontaminated data was opened in EEGLAB, electrode locations and event timing and classifications were saved into an EEG dataset per session and participant. Next, in the ERPLAB package, events were assigned into 4 bins, corresponding to the 4 classifications, and were baseline corrected, averaged, and plotted, both as a temporal series, and a spatial series across the scalp for the P3 wave. Baseline correction was used to eliminate any overall voltage offset from the ERP waveforms in each epoch by subtracting the mean prestimulus voltage in the $100 \mathrm{~ms}$ immediately preceding the FLERP. Finally, the P3 component amplitude was computed in ERPLAB software using the ERP measurement function, which measures the peak amplitude of the $3^{\text {rd }}$ positive peak in the ERP, at $300 \pm 25 \mathrm{~ms}$.

\section{Statistical analysis}

Changes in classification rate, mean reaction times (RT), number of fixations on target, and fixation durations were analyzed before and after training with repeated measures t-test in SPSS 18 software (IBM, Armonk, NY), with significance set at 0.05 . Each of the 4 classifications pre-training was tested separately against the same classification post-training, using weighted means to prevent confounding. The ERP waveforms were analyzed using the ERPLAB 
Journal of Eye Movement Research $6(4): 5,1-11$
Winslow, B., Carpenter, A., Flint, J., Wang, X., Tomasetti, D., Johnston, M. \& Hale, K (2013) Combining EEG and Eye Tracking: Using Fixation-Locked Potentials in Visual Search measurement function, followed by analysis using a repeated measures t-test in SPSS, with significance set at 0.05. The mean amplitude of the P3 component of the ERP was also analyzed across classifications and electrode sites using a one-way ANOVA in SPSS, with significance set at 0.05 .

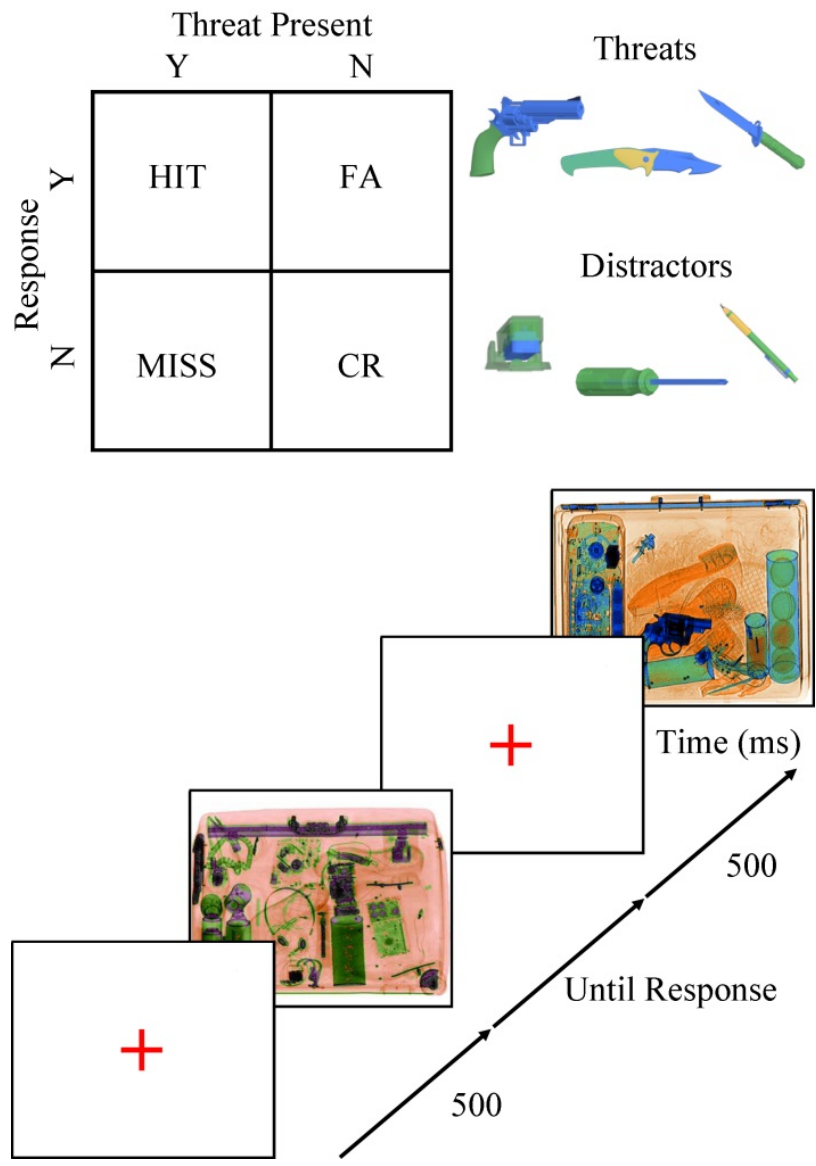

Figure 2 -Participants responded that threats were present by clicking on them with the mouse. When threats were present the trial was classified as a hit, and when they were not present they were classified as a false alarm (FA). Participants indicated no threat by pressing the spacebar key. In this case if no threat was present the trial was classified as a correct rejection (CR), and when a threat was present the trial was classified as a miss.

\section{Results}

\section{Performance}

Following the initial pre-training test session, reaction times and classification rates improved steadily over the first 4 testing sessions, but did not improve over the $5^{\text {th }}$ through $7^{\text {th }}$ testing sessions (see Figure 3). Since the goal of this training exercise is to reach criterion performance, only the pre-training and $4^{\text {th }}$ testing session are presented below.

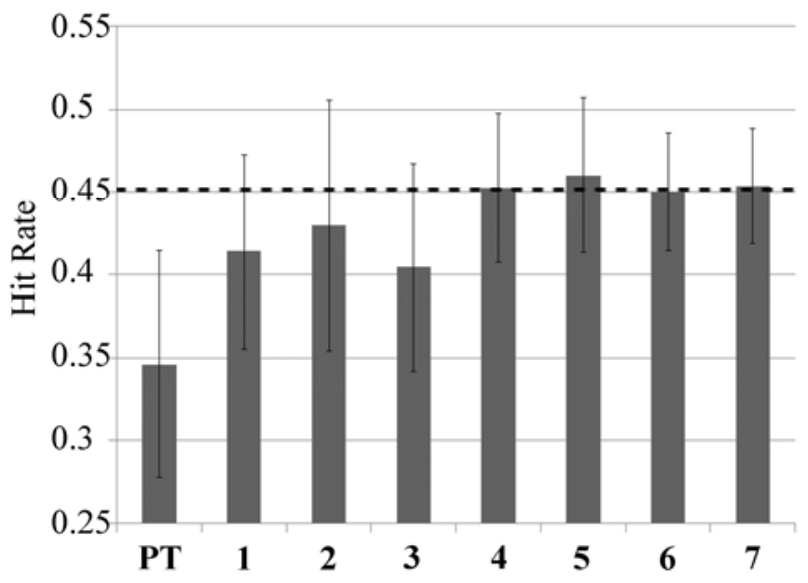

Figure 3 - The average hit rate increased following the pretesting session (PT) through the first 3 testing sessions, then did not change between sessions 4 to 7 .

The mean rates and SD for each condition are shown in Figure 4. The hit rate significantly increased $[T(39)=-$ $8.12, \mathrm{p}<.001]$ by an average of $10 \%$ across all participants following training, and the miss rate significantly decreased $[\mathrm{T}(39)=8.12, \mathrm{p}<.001]$ by $11 \%$. The FA rate significantly increased $[\mathrm{T}(39)=-6.66$, $\mathrm{p}<.001]$ by $5 \%$, and the CR rate significantly decreased $[\mathrm{T}(39)=6.66, \mathrm{p}<.001]$ by $4 \%$. 
Journal of Eye Movement Research $6(4): 5,1-11$
Winslow, B., Carpenter, A., Flint, J., Wang, X., Tomasetti, D., Johnston, M. \& Hale, K (2013) Combining EEG and Eye Tracking: Using Fixation-Locked Potentials in Visual Search

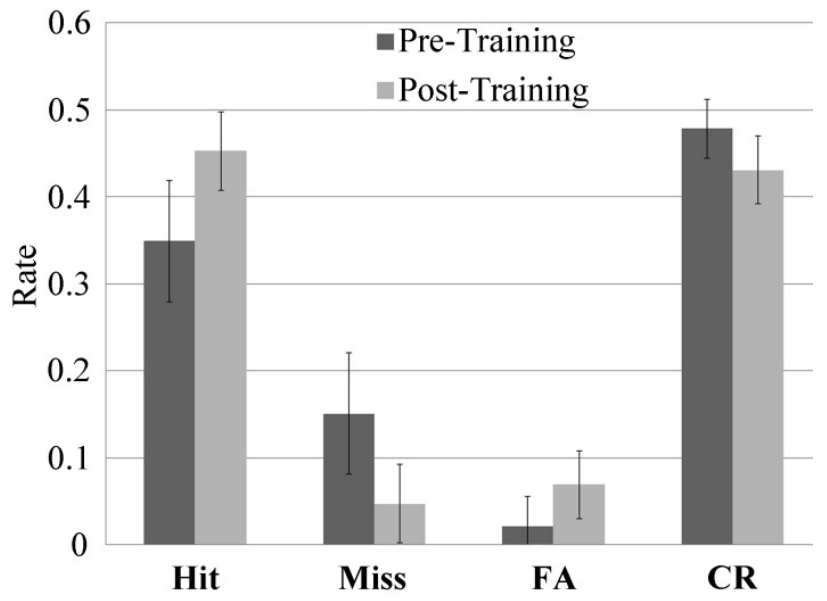

Figure 4 - The mean rates $\pm S D$ for all classifications. All classifications were significantly different following training. The hit rate and $F A$ rate significantly increased. The miss rate and $C R$ rate significantly decreased following training; $p \leq$ 0.001 . Pre-training $d^{\prime}=1.632$; post-training $d^{\prime}=1.364$.

The average reaction time, defined as the time between the presentation of an image and user response, for both pre-training and post-training sessions is shown in Table 1. The average reaction times (RT) decreased significantly for the hit $[\mathrm{T}(39)=6.66, \mathrm{p}<.001]$, miss $[\mathrm{T}(36)=10.47, \mathrm{p}<.001]$, and $\mathrm{CR}[\mathrm{T}(38)=5.39, \mathrm{p}<.001]$ classifications, but not the FA. The interaction effect between training and classification was analyzed via ANOVA, and was not significant, $F(3,7)=2.74, p>0.05$.

Table 1

Mean reaction times in seconds and standard deviations (SD) for the four classifications from all users. The hit, miss, and CR reaction times were significantly different following training. * $p \leq 0.001$.

\begin{tabular}{ccc}
\hline Classification & $\begin{array}{c}\text { Mean RT Pre- } \\
\text { Training (SD) sec }\end{array}$ & $\begin{array}{c}\text { Mean RT Post- } \\
\text { Training (SD) sec }\end{array}$ \\
Hit & $5.42(2.35)$ & $2.85(0.97)^{*}$ \\
Miss & $2.75(0.73)$ & $1.49(0.28)^{*}$ \\
FA & $4.55(3.03)$ & $3.34(1.48)$ \\
CR & $6.86(3.05)$ & $4.09(1.77)^{*}$ \\
\hline
\end{tabular}

\section{Eye Fixations}

The mean number of fixations per threat/distractor is shown in Table 2. Following the training sessions, the average number of fixations per threat/distractor decreased significantly for the hit $[\mathrm{t}(39)=8.68, \mathrm{p}<.001]$, and $\mathrm{CR} \quad \mathrm{T}(39)=2.38, \mathrm{p}=.03]$, classifications. The interaction effect between training and classification was analyzed via ANOVA, and was not significant, $\mathrm{F}(3,7)=3.19, \mathrm{p}>0.05$.

Table 2 - Mean number of fixations and standard deviations (SD) for the four classifications from user responses. The mean number of fixations per threat for the hits and $C R$ were significantly different following training. * $p \leq 0.05$; ** $p \leq 0.001$.

\begin{tabular}{ccc}
\hline Classification & Mean Fixations & Mean Fixations Post- \\
& Pre-Training (SD) & Training (SD) \\
Hit & $9.36(1.89)$ & $6.33(1.18)^{* *}$ \\
Miss & $18.33(6.75)$ & $15.38(5.82)$ \\
FA & $20.05(8.64)$ & $11.18(5.22)$ \\
CR & $17.51(5.27)$ & $14.47(5.31)^{*}$ \\
\hline
\end{tabular}

The increased hit rate was also accompanied by a decrease in the mean fixation duration, defined as the time between the first fixation on threat and participant response, only on threats classified as hit $[\mathrm{T}(39)=6.61$, $\mathrm{p}<.001]$, as shown in Figure 5. Significant changes in mean fixation duration were not seen across the other classifications.

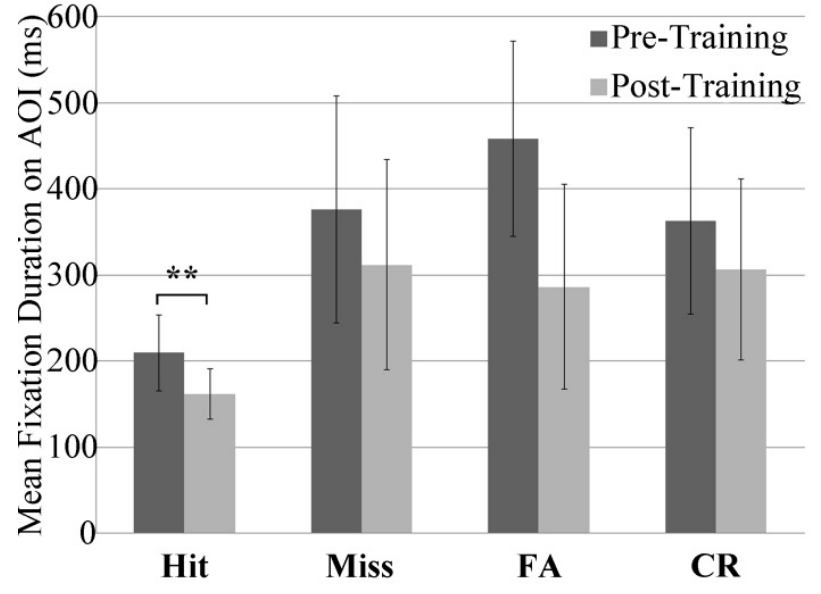

Figure 5 - The mean fixation duration on threats/distractors \pm SD for all classifications. The mean fixation duration decreased significantly following training for trials classified as hits. ${ }^{* *} p \leq 0.001$. 
Journal of Eye Movement Research $6(4): 5,1-11$
Winslow, B., Carpenter, A., Flint, J., Wang, X., Tomasetti, D., Johnston, M. \& Hale, K (2013)

Combining EEG and Eye Tracking: Using Fixation-Locked Potentials in Visual Search

\section{FLERPS}

The average FLERPs for each classification are shown in Figure 6 for the $\mathrm{Pz}$ and $\mathrm{Cz}$ electrode both before and after training. Zero on the $\mathrm{x}$ axis indicates the start of the fixation event. Very little change was seen in the FLERPs before and after training for the hit classification at these sites. Amplitudes were high for the FA classification, due to the relatively low likelihood of this classification event. Notably, there was a large P3 wave seen in the miss classification post-training which was not observed in the pre-training scenario.

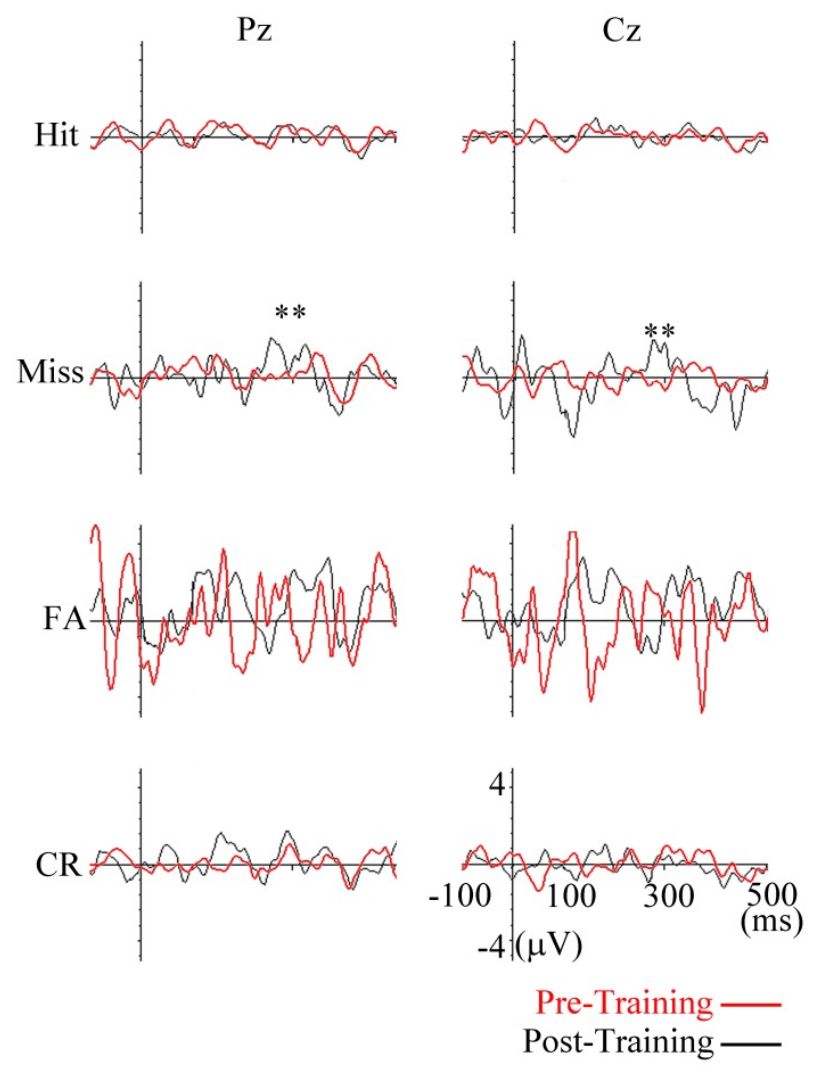

Figure 6-FLERPs at electrodes $P z$ and $C z$, shown as average amplitude $(\mu \mathrm{V})$ over time ( $\mathrm{ms})$. The mean amplitudes of the P3 wave were analyzed using the ERP measurement tool in the ERPLAB package. A significant difference (**p $\leq 0.001)$ was only found for the miss classification.

When the mean amplitude of this component was analyzed, a significant difference $[\mathrm{T}(55)=3.48 ; \mathrm{p}<$ $0.001]$ was found between the pre-training and post- training conditions. When all electrode sites were included, no additional significant differences were observed in the $\mathrm{P} 3$ component following training. A oneway ANOVA was used to test for P3 amplitude differences among the four classifications before and following training. In the pre-training condition, P3 amplitude differed significantly across the four classifications, $[\mathrm{F}(3,32)=8.24, \mathrm{p}<.001]$. Tukey post-hoc comparisons of the four groups indicate that the $\mathrm{CR}$ classification $(\mathrm{M}=0.43,95 \%$ CI $[0.28,0.58])$ was significantly different from the hit $(\mathrm{M}=0.11,95 \%$ CI [$0.07,0.29]), \mathrm{p}<0.05$, and the miss classification $(\mathrm{M}=-$ $0.15,95 \%$ CI $[-0.65,0.05]), \mathrm{p}<0.001$. Comparisons between the other 2 classifications were not statistically significant at $\mathrm{p}<.05$. In the post-training condition, $\mathrm{P} 3$ amplitude differed significantly across the four classifications, $[\mathrm{F}(3,32)=7.33, \mathrm{p}<.001]$. Tukey post-hoc comparisons of the four groups indicate that the hit classification $(\mathrm{M}=0.06,95 \%$ CI $[-0.08,0.20])$ was significantly different from the miss $(\mathrm{M}=1.00,95 \% \mathrm{CI}$ $[0.67,1.33]), \mathrm{p}<0.001$ and the FA classification $(\mathrm{M}=0.76$, $95 \%$ CI $[0.19,1.32]), \mathrm{p}<0.05$, and that the miss classification was significantly different from the CR classification $(\mathrm{M}=0.40,95 \%$ CI $[0.19,0.61]), \mathrm{p}<0.05$. Comparisons between the other 2 classifications were not statistically significant at $\mathrm{p}<.05$, shown in Figure 7 .
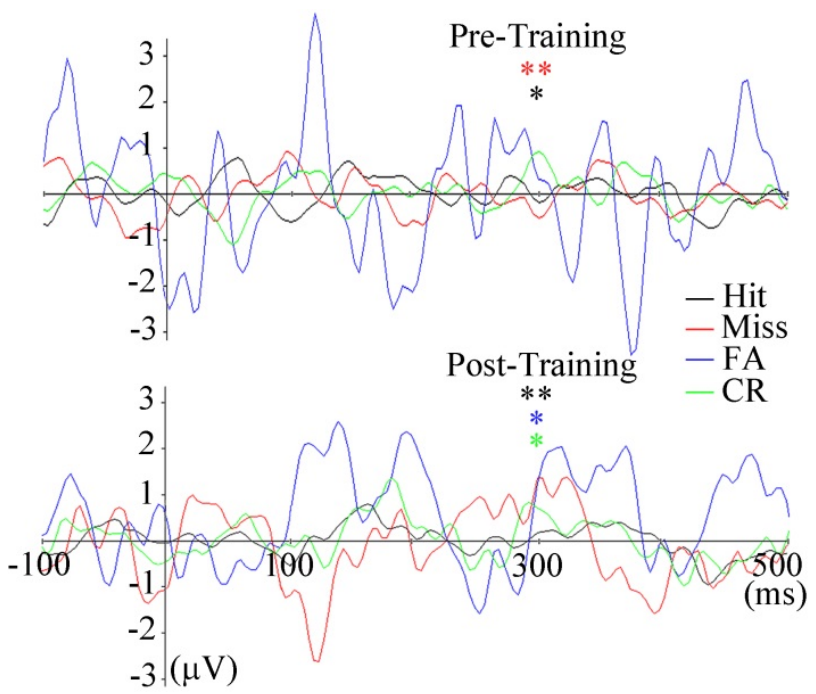
Journal of Eye Movement Research $6(4): 5,1-11$
Winslow, B., Carpenter, A., Flint, J., Wang, X., Tomasetti, D., Johnston, M. \& Hale, K (2013) Combining EEG and Eye Tracking: Using Fixation-Locked Potentials in Visual Search
Figure 7 -Analysis of variance of the P3 components in the pre-training condition uncovered significant differences between the $C R$ and hit classifications $(* p \leq 0.05)$ and the $C R$ and miss classifications $\left({ }^{* *} p \leq 0.001\right)$. In the post-training condition, a statistically significant difference between the hit and miss classification $(* * \leq 0.001)$, between the hit and $F A$ classification $(* p \leq 0.05)$, and between the miss and $C R$ classification $\left({ }^{*} p \leq 0.05\right)$ was found.

\section{Discussion}

The current study demonstrates that fixations obtained from an eye tracker can be used to parse and obtain meaningful insight into the decision making process from the EEG in the time domain. The resulting FLERPs are expected to be able to function as unique neurophysiological signatures to mitigate errors in visual search training due to the statistical differences found within group averages. The current study focused on a single component of the ERP, the P3 wave, but a combination of additional features is expected to significantly increase the performance of the classifier (Dornhege, Blankertz, Curio, \& Müller, 2004). Recently, other groups have reported the utility of combining EEG and eye tracking to assess simple visual search performance (Hale, Fuchs, Axelsson, Baskin, \& Jones, 2007; Kamienkowski, Ison, Quiroga, \& Sigman, 2012), correct artifacts in the EEG (Plochl, Ossandon, \& Konig, 2012), study the process of reading (Dimigen, Sommer, Hohlfeld, Jacobs, \& Kliegl, 2011) and in neuromarketing efforts (Khushaba, Wise, Kodagoda, Louviere, Kahn, \& Townsend, 2013). The current study has shown that even in cluttered imagery such as X-ray scans of carry-on baggage, unique ERP signatures can be obtained from combining eye tracking and EEG to improve performance in scanning tasks.

In this study, significant changes were observed in scan performance, overall performance, and FLERPs following training. Mean reaction times improved for all classifications following training, although due to a difference between the response types (mouse click for threat, spacebar press for clear), small differences in reaction times may be introduced. Distractors were chosen such that they mimicked the size and shapes of knife and gun threats. The resulting FLERPs may indicate different neurophysiological processes, in the case of a threat it may indicate the preparation or execution of a manual response, and in the case of a distractor, it may indicate either the preparation or execution of a key stroke or to continue searching. Additionally, hit rate increased while the mean fixation duration on threats decreased, showing improvements in both accuracy and efficiency in finding and correctly classifying threats due to training. Training sessions also caused a decrease in misses, which is especially critical in baggage screening tasks. Unexpectedly, an increase was seen in FA, expected to reduce overall efficiency in the baggage scanning scenario, along with a concomitant decrease in CR. Previous groups studying visual search scenarios have shown that if targets are highly prevalent, "target present" decisions are more likely and "targetabsent" responses are slower since such a response often leads to a mistake (Wolfe, et al., 2005). Such behavior was observed in this study, as threat prevalence was very high, set to $50 \%$ throughout the scenarios. As seen in the changes to mean reaction times, ERPs, and fixation times, significant learning occurred following training. Such learning is expected to cause changes to both visual search patterns (Sireteanu \& Rettenbach, 2000), as well as plasticity in visual search pathways as participants develop expertise (Walsh, Ellison, Ashbridge, \& Cowey, 1999).

Eye movements introduce artifacts into the EEG, including corneo-retinal dipole changes due to large ocular movements, saccadic spike potentials, and artifacts due to blinking (Plochl, et al., 2012), which are expected to contribute to the variability within an EEG dataset. Baseline correction was applied using the 100 ms of EEG data prior to the start of a fixation event, which included the saccade, and would be prone to artifacts, especially at electrode locations adjacent to the eye. In addition, the changes we observed in this study were in group averages, not specific to single participants. A classifier built upon data averaged from a group is not expected to generalize universally across many subjects due to differences resulting from a wide range of variables such as sensor placement, expertise level, or underlying neurophysiological differences (Del, Mourino, Franze, Cincotti, Varsta, Heikkonen, \& 
Journal of Eye Movement Research $6(4): 5,1-11$
Winslow, B., Carpenter, A., Flint, J., Wang, X., Tomasetti, D., Johnston, M. \& Hale, K (2013) Combining EEG and Eye Tracking: Using Fixation-Locked Potentials in Visual Search
Babiloni, 2002). The EEG system and eye tracker were chosen for this study based upon their wireless capability, low profile, fast setup time, and low cost, making them more likely to be deployed in training scenarios. In addition, due to the relatively low number of electrodes in our EEG system (Srinivasan \& Tucker, 1998), as well as the relatively low sample rates of the EEG system and eye tracker, higher fidelity classifiers could be developed with higher electrode density and sampling rates (Ryynanen, Hyttinen, \& Malmivuo, 2006), at the expense of reducing user comfort and classifier speed due to increased input. The low frequency of the eye tracker also affects the measured durations and latencies, making it difficult to distinguish fixations from other eye movements (Andersson, Nystrom, \& Holmqvist, 2010).

\section{Conclusions}

In summary, by combining a low cost eye tracker and EEG system, significant differences in neurophysiological markers indicative of user decisions were found within a complex X-ray visual search task. Future work is focused on using a combination of features extracted from the EEG to develop and test a classifier to prevent errors in visual search scenarios.

\section{Acknowledgements}

This material is based upon work supported in part by the Department of Homeland Security under BAA HSHQDC-10-C-00213 and SBIR contract D11PC20053. Any opinions, findings, and conclusions or recommendations expressed in this material are those of the authors and do not necessarily reflect the views nor the endorsement of DHS.

\section{References}

Albright, T. D. (2012). On the perception of probable things: neural substrates of associative memory, imagery, and perception. Neuron, 74(2), 227-245.

Andersson, R., Nystrom, M., \& Holmqvist, K. (2010). Sampling frequency and eye-tracking measures: how speed affects durations, latencies, and more. $J$ Eye Mov Res, 3(3), 1-12.

Berka, C., Levendowski, D. J., Cvetinovic, M. M., Petrovic, M. M., Davis, G., Lumicao, M. N., Zivkovic, V. T., Popovic, M., \& Olmstead, R. (2004). Real-time analysis of eeg indexes of alertness, cognition, and memory acquired with a wireless EEG headset. International Journal of Human-Computer Interaction, 17(2), 151-170.

Censor, N., Sagi, D., \& Cohen, L. G. (2012). Common mechanisms of human perceptual and motor learning. Nature Reviews Neuroscience, 13(9), 658-664.

Clark, V. P., Fan, S., \& Hillyard, S. A. (1995). Identification of early visually evoked potential generators by retinotopic and topographic analyses. Human Brain Mapping, 2, 170-187.

Colrain, I. M. (2011). Sleep and the brain. Neuropsychological Review, 21(1), 1-4.

Davidson, P. R., Jones, R. D., \& Peiris, M. T. (2007). EEG-based lapse detection with high temporal resolution. IEEE Transactions on Biomedical Engineering, 54(5), 832-839.

Del, R. M. J., Mourino, J., Franze, M., Cincotti, F., Varsta, M., Heikkonen, J., \& Babiloni, F. (2002). A local neural classifier for the recognition of EEG patterns associated to mental tasks. IEEE Transactions on Neural Networks, 13(3), 678-686.

Delorme, A., \& Makeig, S. (2004). EEGLAB: an open source toolbox for analysis of single-trial EEG dynamics including independent component analysis. Journal of Neuroscience Methods, 134(1), 9-21. 
Journal of Eye Movement Research $6(4): 5,1-11$
Winslow, B., Carpenter, A., Flint, J., Wang, X., Tomasetti, D., Johnston, M. \& Hale, K (2013) Combining EEG and Eye Tracking: Using Fixation-Locked Potentials in Visual Search
Dimigen, O., Sommer, W., Hohlfeld, A., Jacobs, A. M., \& Kliegl, R. (2011). Coregistration of eye movements and EEG in natural reading: analyses and review. Journal of Exerimental Psychology, General, 140(4), 552-572.

Dornhege, G., Blankertz, B., Curio, G., \& Müller, K. R. (2004). Boosting bit rates in noninvasive EEG single-trial classifications by feature combination and multiclass paradigms. IEEE Transactions on Biomedical Engineering, 51(6), 993-1002.

Duchowski, A. T. (2002). A breadth-first survey of eye-tracking applications. Behavior Reseach Methods Instruments \& Computers, 34(4), 455470.

Hale, K. S., Fuchs, S., Axelsson, P., Baskin, A., \& Jones, D. (2007). Determining gaze parameters to guide EEG/ERP evaluation of imagery analysis. In D. D. Schmorrow, D. M. Nicholson, J. M. Drexler \& L. M. Reeves (Eds.), Foundations of Augmented Cognition (4th Edition). Arlington, VA: Strategic Analysis, Inc.

Hansen, D. W., \& Ji, Q. (2010). In the eye of the beholder: a survey of models for eyes and gaze. IEEE Transactions on Pattern Analysis and Machine Intelligence, 32(3), 478-500.

Hillyard, S. A., Vogel, E. K., \& Luck, S. J. (1998). Sensory gain control (amplification) as a mechanism of selective attention: electrophysiological and neuroimaging evidence. Philosophical Transactions of the Royal Society of London Series B-Biological Sciences, 353(1373), 1257-1270.

Jacob, R. J. K. (1991). The use of eye movements in human-computer interaction techniques: what you look at is what you get. ACM Transactions on Information Systems, 9(2), 152-169.

Kamienkowski, J. E., Ison, M. J., Quiroga, R. Q., \& Sigman, M. (2012). Fixation-related potentials in visual search: a combined EEG and eye tracking study. Journal of Vision, 12(7), 4.
Kastner, S., \& Ungerleider, L. G. (2000). Mechanisms of visual attention in the human cortex. Annual Review of Neuroscience, 23, 315-341.

Khushaba, R. N., Wise, C., Kodagoda, S., Louviere, J., Kahn, B. E., \& Townsend, C. (2013). Consumer neuroscience: assessing the brain response to marketing stimuli using electroencephalogram (EEG) and eye tracking. Expert Systems with Applications, 40(9), 3803-3812.

Koch, C., \& Ullman, S. (1985). Shifts in selective visual attention: towards the underlying neural circuitry. Human Neurobiology, 4(4), 219-227.

Lagerlund, T. D., Cascino, G. D., Cicora, K. M., \& Sharbrough, F. W. (1996). Long-term electroencephalographic monitoring for diagnosis and management of seizures. Mayo Clinic Proceedings, 71(10), 1000-1006.

Landolt, H. P. (2011). Genetic determination of sleep EEG profiles in healthy humans. Progress in Brain Research, 193, 51-61.

Macmillan, N. A., \& Creelman, C. D. (2004). Detection theory: a user's guide (2nd edition), Psychology Press, Mahwah NJ. Mahwah, NJ.

Mendez, O. E., \& Brenner, R. P. (2006). Increasing the yield of EEG. J Clin Neurophysiol, 23(4), 282293.

Montagna, B., Pestilli, F., \& Carrasco, M. (2009). Attention trades off spatial acuity. Vision Research, 49(7), 735-745.

Mulckhuyse, M., \& Theeuwes, J. (2010). Unconscious attentional orienting to exogenous cues: A review of the literature. Acta Psychologica, 134(3), 299-309.

Müller, H. J., \& Krummenacher, J. (2006). Visual search and selective attention. Visual Cognition, 14(4-8), 389-410. 
Journal of Eye Movement Research $6(4): 5,1-11$
Winslow, B., Carpenter, A., Flint, J., Wang, X., Tomasetti, D., Johnston, M. \& Hale, K (2013) Combining EEG and Eye Tracking: Using Fixation-Locked Potentials in Visual Search
Naatanen, R., \& Picton, T. (1987). The N1 wave of the human electric and magnetic response to sound: a review and an analysis of the component structure. Psychophysiology, 24(4), 375-425.

Petersen, S. E., \& Posner, M. I. (2012). The attention system of the human brain: 20 years after. Annual Review of Neuroscience, 35, 73-89.

Plochl, M., Ossandon, J. P., \& Konig, P. (2012). Combining EEG and eye tracking: identification, characterization, and correction of eye movement artifacts in electroencephalographic data. Frontiers in Human Neuroscience, 6, 278.

Ryynanen, O. R., Hyttinen, J. A., \& Malmivuo, J. A. (2006). Effect of measurement noise and electrode density on the spatial resolution of cortical potential distribution with different resistivity values for the skull. IEEE Transactions on Biomedical Engineering, 53(9), 1851-1858.

Salvucci, D. D., \& Goldberg, J. H. (2000). Identifying fixations and saccades in eye-tracking protocols Proceedings of the Eye Tracking Research and Applications Symposium (pp. 71-78). New York: ACM Press.

Sireteanu, R., \& Rettenbach, R. (2000). Perceptual learning in visual search generalizes over tasks, locations, and eyes. Vision Research, 40(21), 2925-2949.

Song, Y., Ding, Y., Fan, S., \& Chen, L. (2002). An event-related potential study on visual perceptual learning under short-term and long-term training conditions. Neuroreport, 13(16), 2053-2057.

Srinivasan, R., \& Tucker, D. M. (1998). The spatial Nyquist of the human EEG. Behavior Reseach Methods Instruments \& Computers, 30(1), 8-19.

Stickgold, R., Whidbee, D., Schirmer, B., Patel, V., \& Hobson, J. A. (2000). Visual discrimination task improvement: A multi-step process occurring during sleep. Journal of Cognitive Neuroscience, 12(2), 246-254.
Sutton, S., Braren, M., Zubin, J., \& John, E. R. (1965). Evoked-potential correlates of stimulus uncertainty. Science, 150(3700), 1187-1188.

Treisman, A. (2006). How the deployment of attention determines what we see. Visual Cognition, 14(4-8), 411-443.

Treisman, A., \& Gelade, G. (1980). A featureintegration theory of attention. Cognitive Psychology, 12(1), 97-136.

Wada, M. (1999). Measurement of olfactory threshold using an evoked potential technique. Rhinology, 37(1), 25-28.

Walsh, V., Ellison, A., Ashbridge, E., \& Cowey, A. (1999). The role of the parietal cortex in visual attention--hemispheric asymmetries and the effects of learning: a magnetic stimulation study. Neuropsychologia, 37(2), 245-251.

Wickens, C. D., Hollands, J. G., Banbury, S., \& Parasuraman, R. (2012). Engineering psychology and human performance (4th edition), Pearson, Upper Saddle River, NJ. Columbus, OH.

Wolfe, J. M., Horowitz, T. S., \& Kenner, N. M. (2005). Cognitive psychology: rare items often missed in visual searches. Nature, 435(7041), 439440.

Wolfe, J. M., \& Van Wert, M. J. (2010). Varying target prevalence reveals two dissociable decision criteria in visual search. Current Biology, 20(2), 121-124. 